\title{
Friedel Oscillations in Carbon Nanotube Quantum Dots and Superlattices
}

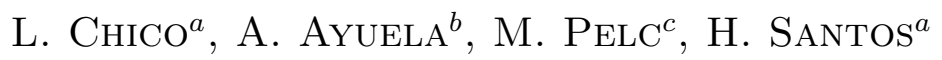 \\ AND W. JASKÓLSKI ${ }^{c}$ \\ ${ }^{a}$ Departamento de Física Aplicada UCLM, 45071 Toledo, Spain \\ ${ }^{b}$ Centro Mixto CSIC-UPV/EHU, 20080 Donostia, Spain \\ ${ }^{c}$ Instytut Fizyki UMK, Grudziạdzka 5, 87-100 Toruń, Poland
}

Interface states of all-metallic carbon nanotube quantum dots and superlattices are studied within a tight-binding model. We focus on achiral systems made by connecting armchair $(n, n)$ and zigzag $(2 n, 0)$ tubes with a full ring of $n$ pentagon-heptagon topological defects. We show that the coupling between interface states, which arise from the topological defects, reflects the existence of the Friedel oscillations in the $(n, n)$ tube, with an unusually large decay exponent. We expect this interaction to be important for the understanding of other physical properties, such as selective dot growth, magnetic interaction through carbon tubes or optical spectroscopy of interface states.

PACS numbers: 73.22.-f, 73.90+f

\section{Introduction}

Carbon nanotubes are one of the most promising materials for nanotechnological applications [1]. A variety of nanodevices entirely based on carbon nanotubes have been demonstrated [2], exploiting the unique relation between geometry and electronic properties shown by these systems. Since carbon nanotubes can behave as semiconductors or metals, the proposal of structures made by joining different kinds of tubes with topological defects has an evident interest for the design of one-dimensional devices. Therefore, the electronic structure of single junctions [3], quantum dots $[4,5]$ and superlattices $[6,7]$ has been the subject of many theoretical investigations. Recently, the controlled growth of carbon nanotube intramolecular junctions by temperature variations has been reported [8]. This breakthrough may open the possibility of fabricating complex nanotube devices, which have to be fully characterized. Most of the theoretical effort is focused on states due to quantum confinement. However, due to the presence of topological defects, these systems may have interface states, which have a profound influence in their electronic properties and performance. These interface states have not been so thoroughly analyzed, and they are the focus of the present work. 
We have studied the interface states of achiral carbon nanotube quantum dots and superlattices within a $\pi$-orbital tight-binding model. We focus on metallic-based structures made by connecting armchair $(n, n)$ tubes with zigzag $(2 n, 0)$ tubes, with $n$ multiple of three. Junctions between these tubes contain $n$ pentagon-heptagon pairs that give rise to interface states when $n>3$. With a double junction, a quantum dot (QD) can be formed: The $N(2 n, 0)$ QDs are made sandwiching $N$ unit cells of a $(2 n, 0)$ tube between two semiinfinite $(n, n)$ leads; we denote such structures as $(n, n) / N(2 n, 0) /(n, n)$ or, more briefly, $N(2 n, 0)$. Conversely, a $N(n, n) \mathrm{QD}$ is made by joining an $N(n, n)$ portion to two $(2 n, 0)$ leads, making a $(2 n, 0) / N(n, n) /(2 n, 0)$ structure. Superlattices (SLs) consist of a periodic sequence of $N(2 n, 0)$ and $M(n, n)$ portions of nanotubes joined by $n$ pentagon-heptagon pairs, denoted as $M(n, n) / N(2 n, 0)$. We calculate QD spectra using a Green function matching technique; for SLs direct diagonalization provides bands and their corresponding wave functions.
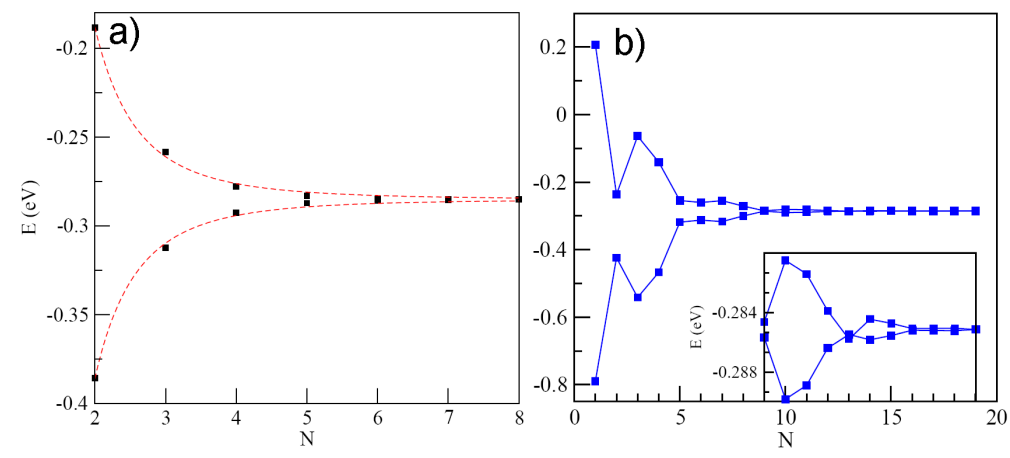

Fig. 1. (a) Energies of the interface states of the $(6,6) / N(12,0) /(6,6)$ quantum dots vs. dot size $N$. The dashed lines show the fits to $\propto 1 / d^{\alpha}$. (b) Interface state energies for the $(12,0) / N(6,6) /(12,0)$ dots as a function of dot size $N$. The inset in (b): zoom in the region of large $N$ for the $N(6,6)$ series.

The bands of armchair and zigzag tubes close to the Fermi energy have different symmetry; this symmetry barrier gives rise to confinement in multiple junction structures based on these kinds of tubes. Thus, completely localized states appear in QDs [5] and dispersionless bands are found in SLs [6, 7]. However, in this work we focus on the nature of interface states, which are due to topological defects at the junction between tubes and therefore have a mixed character. In single junctions interface states are well localized and easy to identify because of their high local density of states (LDOS), but in short-period QDs or SLs a more detailed symmetry analysis is needed to identify them [7].

\section{Results and discussion}

In short-period SLs or QDs of small size, interface states interact and their energies split and differ considerably from the energy of interface state at the single 

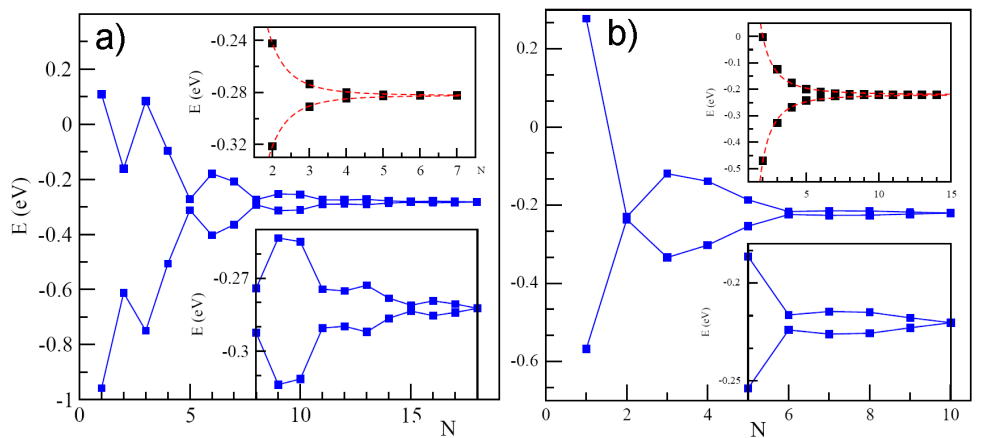

Fig. 2. Interface state energies for the $N(9,9)$ and $N(18,0)$ (upper insets) quantum dots vs. dot size $N$. Part (a) corresponds to the series converging to $E_{i 1}=-0.2824 \mathrm{eV}$ in the limit of large $N$; (b) shows the series converging to $E_{i 2}=-0.2203 \mathrm{eV}$. The dashed lines in the upper insets show the fits to $\propto 1 / d^{\alpha}$. Lower insets: zoom in the region of large $N$ for the corresponding $N(9,9)$ series.

junction $[6,5]$. As expected, in the limit of large sizes, their energies converge to the single junction limit and they are mainly localized in the defect interface region. Surprisingly, the energy dependence with system size depends on the geometry: for QDs, we find that zigzag $N(2 n, 0)$ dots show a monotonous behavior, whereas for $N(n, n)$ armchair QDs, the interface state energies oscillate with the system size $N$. We can check this in two specific examples: In Fig. 1 we show the energies of the interface states vs. dot size for the $N(12,0)$ and $N(6,6)$ cases. Figure 1a depicts the interface state energies for the zigzag $N(12,0)$ QD series, with a clear monotonic behavior. In Fig $1 b$ the $N(6,6)$ QD interface energies present clear oscillations; the inset zooms the data for larger $N$. We see in all cases that the energies converge to the interface energy of the single junction, $E_{i}=-0.2850 \mathrm{eV}$. A larger radii case, the $(18,0)$ and $(9,9)$ QDs, is shown in Fig. 2. As the single $(18,0) /(9,9)$ junction has two interface states with energies $E_{i 1}=-0.2824 \mathrm{eV}$ and $E_{i 2}=-0.2203 \mathrm{eV}$, for these QDs we have to analyze two series of levels, corresponding to $E_{i 1}$ and $E_{i 2}$, shown in Fig. $2 \mathrm{a}$ and b, respectively. We find the same behavior, i.e., a monotonous decay for the zigzag QD series, displayed in the upper insets, and an oscillating pattern for the armchair QD series.

This is also observed for superlattices. In order to compare SLs bands with QD states, we plot the energy of the band center for the SL cases. In Fig. 3 we present the superlattice series corresponding to the QDs shown in Fig. 1: i.e., the $M(6,6) / N(12,0)$ SL interface bands, whereas Fig. 4 displays the results for the $M(9,9) / N(18,0)$ SLs. The cases selected correspond to a sufficiently large fixed unit cell length, in order to make a comparison with the QD cases. We can see that for $M(n, n) / N(2 n, 0)$ SL series with fixed $M$ and varying zigzag length, the interface state energy bands have a monotonic variation. However, oscillations are observed if the armchair length $M$ is varied. Let us notice that for the varying 


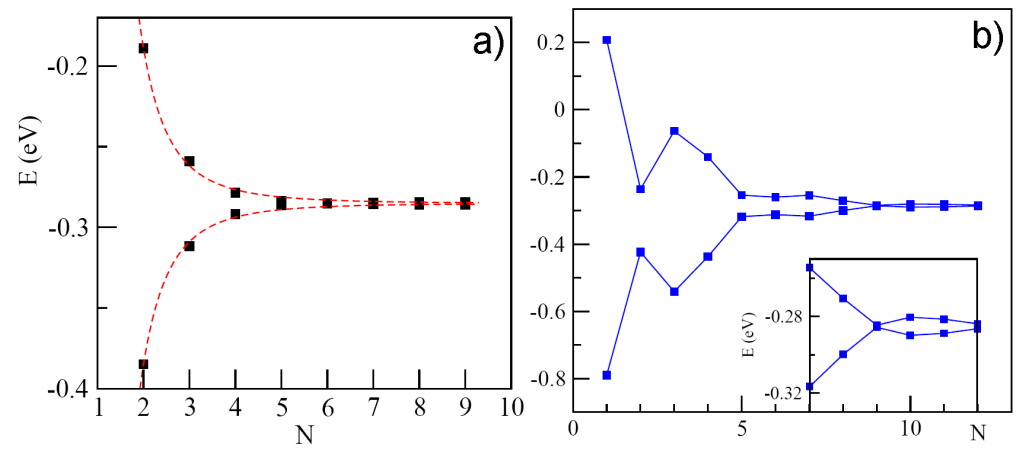

Fig. 3. Energies of the interface bands for the $N(12,0) / 14(6,6)$ (a) and $8(12,0) / N(6,6)$ (b) superlattices vs. $N$. The inset in (b): zoom in the region of large $N$.
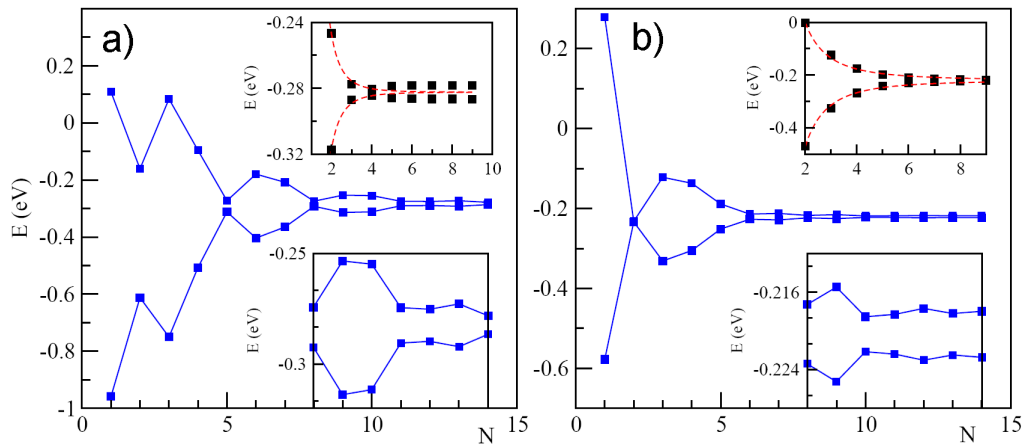

Fig. 4. Interface band energies for the $8(18,0) / N(9,9)$ and $N(18,0) / 14(9,9)$ (upper insets) superlattices vs. dot size $N$. Part (a) corresponds to the series converging to $E_{i 1}=-0.2824 \mathrm{eV}$ in the limit of large $N$; (b) shows the series converging to $E_{i 2}=$ $-0.2203 \mathrm{eV}$. The dashed lines in the upper insets show the fits to $\propto 1 / d^{\alpha}$ for the $N(18,0)$ series. Lower insets: zoom in the region of large $N$ for the corresponding $N(9,9)$ interface band series.

zigzag length series shown in the upper insets, the energies of the higher and lower energy bands do not converge to the same energy value; there is a small splitting due to coupling through the armchair section. In order to fit these zigzag series, we have taken the average of the two limit values; this average actually equals the interface state energy of the single junction. The fits are shown in dashed lines in the upper insets. Due to coupling through the fixed length sections, there are some numerical differences between SL and QD results, but the general trend is the same as shown for quantum dots. Below we show how these apparently disparate behaviors found in zigzag and armchair series can be understood as Friedel-like oscillations.

In general, the Friedel oscillations are related to the presence of impurities, defects or inhomogeneities — such as surfaces — in metallic systems, typically 
reflected in the charge density, but ubiquitous in other properties of the system, such as total energies or magnetic coupling. Effectively, the magnitude of interest presents oscillations that can be described by the function as

$$
f(d)=\frac{A \sin \left(2 k_{\mathrm{F}}\left(d+\delta_{0}\right)\right)}{d^{\alpha}},
$$

where $k_{\mathrm{F}}$ is the wave vector of the metallic host, $\delta_{0}$ is a phase shift that takes into account the wave function penetration, $d$ is the length of the metallic part responsible of the oscillatory behavior, and $\alpha$ is a damping factor usually related to the dimension of the system [9], being $\alpha=3$ for impurities in three-dimensional metals, $\alpha=2$ for two-dimensional systems and so forth. Thus, on the one hand, given that for zigzag metallic tubes $k_{\mathrm{F}}=0$, it can be understood that for $M(2 n, 0)$ QDs, or for SL series with varying zigzag tube length, the behavior is not oscillatory, but monotonous. On the other hand, oscillations are observed in armchair QDs or in SL series with varying armchair length, because in this case is nonzero, $k_{\mathrm{F}} \approx \frac{2}{3} \frac{\pi}{a}$, with $a=2.46 \AA$, the unit cell length of any armchair tube.

To verify, whether these oscillations are Friedel-like, we have studied the energy dependence with system size in the instances described above. We concentrate on the interface state of the lowest energy. In the first place, in order to obtain the damping factor, we focus in the zigzag series, where $k_{\mathrm{F}}=0$. Fitting the data shown in Fig. 1a, we get a value around $\alpha=3.5$ in the $N(12,0)$ series. With respect to the $N(18,0)$ QDs shown in Fig. 2, we obtain the values $\alpha_{1}=3.9$ and $\alpha_{2}=2.5$, respectively. These damping factors are larger than expected for one-dimensional systems, which is given by the system dimension, $\alpha=1$. Another remarkable fact is that different exponents are found for the two interface states in the same system, the $N(18,0)$ series. These states show a quite different degree of confinement, pointing to the fact that besides dimensionality, localization plays an important role.

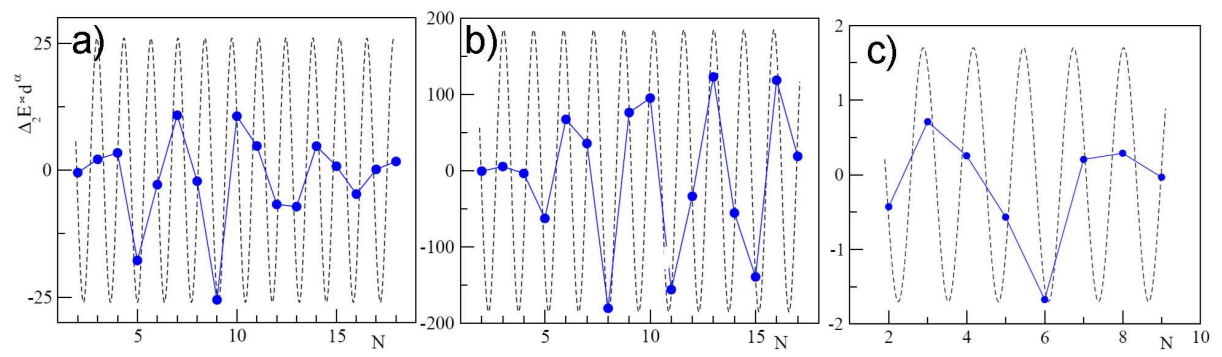

Fig. 5. Second derivative of the lowest interface state energy (full line with circles) multiplied by the dot size $d$ to the $\alpha$ as a function of the dot size $N$. Part (a) shows the results for the $N(6,6)$ quantum dot. Parts (b) and (c) correspond to the two interface state series of the $N(9,9)$ quantum dot; (b) shows the series converging to $E_{i 1}=-0.2824$ $\mathrm{eV}$ in the limit of large $N$, and (c) presents the series converging to $E_{i 2}=-0.2203 \mathrm{eV}$. The dashed lines are the fits to Eq. (1) multiplied by $\propto 1 / d^{\alpha}$. 

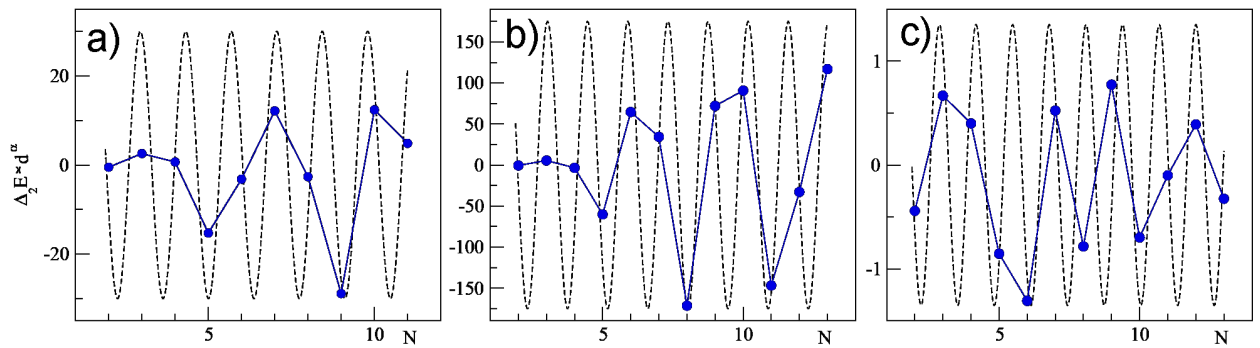

Fig. 6. Second derivative of the lowest interface state energy (full line with circles) multiplied by the dot size $d$ to the $\alpha$ for the $N(6,6) / M(12,0)$ SL $(\mathrm{a}),(9,9) /(18,0)$ series converging to $E_{i 1}=-0.2824 \mathrm{eV}(\mathrm{b})$, and $(9,9) /(18,0)$ series converging to $E_{i 2}=$ $-0.2203 \mathrm{eV}$ (c). The dotted line is the fit to the function defined by Eq. (1) times $d^{\alpha}$.

We use these damping factors to fit the energies of the interface states in the $N(n, n)$ QD series shown in Figs. 1 and 2. As the amplitude of the oscillations decreases with increasing length, it is commonly studied the second derivative of the magnitude of interest, where the oscillations are more evident. Figure 5 shows the second derivative of the lowest interface state energies for the QD cases presented in Figs. 1 and 2, where the damping is removed by multiplying by $d^{\alpha}$. The spatial periods obtained are 1.4 unit cells for the $N(6,6)$ series and 1.4 and 1.3 unit cells for the interface states $E_{i 1}$ and $E_{i 2}$ of the $N(9,9)$ QDs, respectively, close to theoretically expected of $\frac{3}{2}$ unit cells.

The oscillations in SLs have been studied using the same approach. Figure 6a shows the oscillating part of the second derivative of the lowest interface band energy for a $M(12,0) / N(6,6) \mathrm{SL} ;$ Fig. $6 \mathrm{~b}$ and c show the same quantity for the two lowest interface bands series of the $(18,0) /(9,9) \mathrm{SL}$. The spatial periods obtained for the SLs are roughly the same as for QDs, with differences below 5\%. In all cases presented in Figs. 5 and 6 , the oscillations seem to have a larger periodicity; this is the so-called aliasing effect. The interference between the two characteristic lengths of the system, i.e., the system size and the wavelength $\approx\left(2 k_{\mathrm{F}}\right)^{-1}$, gives the impression of a longer periodicity, but, as described above, all the spatial periods obtained are around the theoretically expected value.

The Friedel oscillations in carbon nanotubes may have different consequences: it may govern growth processes in systems involving metallic nanotubes with $k_{\mathrm{F}} \neq 0$, yielding a size selection, as it happens with nanoclusters [10], where magic numbers for cluster sizes appear. It may also influence the magnetic coupling through metallic nanotubes, as it happens in metallic multilayers [11, 12]. Finally, the interface bands of the zigzag/armchair SLs studied here appear just below and above the Fermi level. Thus, the oscillations in level separation will surely be observed in optical experiments.

To summarize, we have shown that interface states and bands in pure metallic carbon nanotube structures may reflect the existence of the Friedel oscillations in 
metallic tubes with $k_{\mathrm{F}} \neq 0$. The oscillations of the energies of interface states may influence physical properties and processes of the carbon nanotube junction systems, as for example system growth.

\section{Acknowledgments}

We acknowledge financial support by the ETORTEK (NANOMAT) program of the Basque government, the Intramural Special Project (Ref. 2006601242), the Spanish Ministerio de Ciencia y Tecnología (Grants No. Fis 2007-66711-C02C01 and MAT2006-06242), JCCM Grant No. PAI08-0067-2673 and the European Network of Excellence NANOQUANTA (NM4-CT-2004-500198).

\section{References}

[1] Carbon Nanotubes: Advanced Topics in the Synthesis, Structure, Properties and Applications, Eds. A. Jorio, G. Dresselhaus, M.S. Dresselhaus, Springer-Verlag, Berlin 2008.

[2] P.G. Collins, A. Zettl, H. Bando, A. Thess, R.E. Smalley, Science 278, 100 (1997); J.U. Lee, P.P. Gipp, G.M. Heller, Appl. Phys. Lett. 85, 145 (2004); S.J. Tans, A.R.M. Verschueren, C. Dekker, Nature (London) 393, 49 (1998).

[3] L. Chico, V.H. Crespi, L.X. Benedict, S.G. Louie, M.L. Cohen, Phys. Rev. Lett. 76, 971 (1996); L. Chico, L.X. Benedict, S.G. Louie, M.L. Cohen, Phys. Rev. B 54, 2600 (1996).

[4] L. Chico, M.P. López Sancho, M.C. Muñoz, Phys. Rev. Lett. 81, 1278 (1998); C.G. Rocha, T.G. Dargam, A. Latgé, Phys. Rev. B 65, 165431 (2002); W. Zhang, W. Lu, E.G. Wang, Phys. Rev. B 72, 075438 (2005).

[5] L. Chico, W. Jaskólski, Phys. Rev. B 69, 085406 (2004).

[6] W. Jaskólski, L. Chico, Phys. Rev. B 71, 155305 (2005).

[7] A. Ayuela, L. Chico, W. Jaskólski, Phys. Rev. B 77, 085435 (2008).

[8] Y. Yao, Q. Li, J. Zhang, R. Liu, L. Jiao, Y.T. Zhu, Z. Liu, Nature Mater. 6, 283 (2007).

[9] R. Egger, H. Grabert, Phys. Rev. Lett. 75, 3505 (1995).

[10] J.A. Alonso, M.J. Lopez, Journal of Cluster Science 14, 31 (2003).

[11] P. Czoschke, H. Hong, L. Basile, T.-C. Chiang, Phys. Rev. Lett. 93, 036103 (2004).

[12] A. Ayuela, E. Ogando, N. Zabala, Phys. Rev. B 75, 153403 (2007). 